\title{
Species-specific detection of $C$. difficile using targeted antibody design
}

\author{
Lawry, B.M ${ }^{1 \#}$.Johnson, C.L ${ }^{2 \#}$., Flanagan, $\mathrm{K}^{3}$., Spoors, J.A. ${ }^{2}$., McNeil, C.J ${ }^{2}$, Wipat, $\mathrm{A}^{3}$., Keegan, $\mathrm{N}^{2 *}$. \\ ${ }^{1}$ School of Biomedical Sciences, Faculty of Medical Sciences, Newcastle University, Newcastle-Upon- \\ Tyne, NE2 4HH, UK \\ ${ }^{2}$ Diagnostic and Therapeutic Technologies, Institute of Cellular Medicine, Newcastle University, \\ Newcastle-Upon-Tyne, NE2 4HH, UK \\ ${ }^{3}$ School of Computing, Urban Sciences Building, Newcastle University, Newcastle-Upon-Tyne, NE4 \\ 5TG, UK \\ \# Both authors contributed equally to this work \\ * Corresponding Author, Email: Neil.Keegan@ncl.ac.uk
}

\section{Abstract}

Clostridium difficile is a Gram-positive, spore-forming bacterium that continues to present a world-wide problem in healthcare settings. The bacterium causes disease, the symptoms of which include diarrhoea, fever, nausea, abdominal pain and even death. Despite the prevalence of the disease, the diagnosis of $C$. difficile infection is still challenging, with a variety of methods available, each varying in their effectiveness. In this work we sought to identify a new biomarker for $C$. difficile, develop affinity reagents and design a diagnostic assay for $C$. difficile infection which could be used in a typical two-step testing algorithm. Initially a bioinformatics pipeline was developed that identified a surface associated biomarker "AKDGSTKEDQLVDALA" present in all $C$. difficile strains sequenced to-date and unique to the $C$. difficile species. Monoclonal antibodies were subsequently raised against peptides corresponding to the biomarker sequence. During characterisation studies, monoclonal antibody 521 (mAb521) was shown to bind all known C. difficile surface layer types, but not closely related strains. Surface plasmon resonance measurements were used to calculate an apparent equilibrium dissociation constant of $36.5 \mathrm{nM}$ between the purified protein target containing the biomarker (surface layer protein A) and mAb521. We demonstrate a limit of detection of $12.4 \mathrm{ng} / \mathrm{ml}$ against surface layer protein $A$ and $1.7 \times 10^{6}$ cells $/ \mathrm{ml}$ in minimally processed $C$. difficile cultures. The utility of this computational approach to antibody design for diagnostic tests is the ability to produce antibodies which can act as universal species identifiers whilst mitigating the likelihood of false-positive detection by intelligently screening potential biomarkers against RefSeq data for other non-target bacteria.

\section{Introduction}

Clostridium difficile infection (CDI) continues to be a significant economic burden, particularly in healthcare settings, with the spectrum of clinical disease ranging from mild diarrhoea to death. C. difficile includes both pathogenic (toxin-producing) and non-pathogenic strains, with 
both strains able to colonise their hosts, however only toxin producing strains are associated with disease ${ }^{1}$.

The choice of laboratory test for $C$. difficile is a contentious one with a multitude of tests available. One commonly used test is an enzyme immunoassay (EIA) which detects glutamate dehydrogenase $(\mathrm{GDH})$, a secreted enzyme found in stool samples during CDI which converts L-glutamate into $\alpha$-ketoglutarate. This pan- $C$. difficile antigen can be tested for in a few hours using the glutamate dehydrogenase enzyme immunoassay (GDH EIA), however it does not determine whether the $C$. difficile strain is actively producing toxin ${ }^{2}$. To elucidate this, the toxins associated with $C D I$, toxin $A / B$, are typically assayed using a second EIA, again typically taking a few hours to process ${ }^{3}$. More recently, nucleic acid amplification tests (NAAT) have been increasingly used for toxin $A / B$ testing ${ }^{4}$. However, when relying solely on molecular methods for CDI diagnosis, it has been suggested this results in the over-diagnosis of CDI through the detection of asymptomatic carriage, resulting in overtreatment and increased healthcare costs ${ }^{5}$. The current protocol for testing and diagnosing CDI in the UK is based on guidelines from the Department of Health, who advise that organisations adhere to a twostep testing algorithm which consists of a GDH EIA (or NAAT) measurement to screen samples, followed by a toxin A/B EIA ${ }^{6}$. If the first test in the algorithm is negative, the second test does not need to be performed. The authors of the guidelines acknowledge that no test or combination of tests is infallible and the clinical condition of the patient should always be taken into consideration when making management and treatment choices.

Rates and severity of CDI in hospitals in Europe and North America have increased since 2000 and correlate with the dissemination of an epidemic strain, 027 , characterised by higher than usual toxin $A / B$ production in addition to demonstrating resistance to fluoroquinolone and cephalosporin antibiotics ${ }^{7}$. Typing studies from 2008 revealed that PCR ribotype 027 was the most common strain isolated from symptomatic patients, accounting for over $41.3 \%$ of isolates in English hospitals, followed by type 106 (20.2\%) and type 001 (7.8\%) ${ }^{8}$. A mixture of 44 other PCR ribotypes accounted for the remaining $28.9 \%$ of isolates ${ }^{8}$. More recent data from the $C$. difficile Ribotyping Network indicate that 027 prevalence has decreased in the United Kingdom, potentially because of efforts to reduce fluoroquinolone and cephalosporin usage ${ }^{9}$.

Although a relatively simple and inexpensive assay, concerns have been raised about the sensitivity of the commonly used GDH EIA, particularly for non-027 strains ${ }^{10}$. This is not because of strain-dependent difference in GDH expression but most likely because 027 strains tend to attain higher organism burdens, which means that GDH is easier to detect using the GDH EIA as it is present at a greater multiplicity ${ }^{11}$. Although the sensitivity of the GDH EIA is comparable to PCR for 027 strains, it has been demonstrated that PCR is significantly more sensitive than the GDH EIA for detecting strains of ribotypes other than $027^{12}$. Another disadvantage of the GDH EIA is that antiserum against $C$. difficile GDH has the potential to cross-react with GDH from other anaerobes ${ }^{3}$. Despite the sensitivity and specificity of the GDH EIA being inferior to PCR it is still widely used in clinical diagnostic algorithms, because it is cheaper and quicker than standard PCR. 
Bearing in mind the current limitations outlined above, we sought to create a new diagnostic test for CDI which could act as an alternative to the GDH EIA. A major hurdle in the development of any new medical diagnostic test for infectious disease is the development of suitable affinity reagents, such as antibodies, that bind specifically to the target organism. To address this challenge, and to find a new specific biomarker for $C$. difficile, a bioinformatics method was developed building on a previously described approach ${ }^{13}$. This approach is able to predict biomarkers for a given group of bacteria from their genome sequence data. In this work we demonstrate the utility of a computational approach for generating species-specific C. difficile monoclonal antibodies (mAbs) against a biomarker ubiquitous to all $C$. difficile strains sequenced to-date.

\section{Materials and Methods}

\section{Biomarker identification in C. difficile using a bioinformatics approach}

There are several idealised requirements for pathogenic biomarkers in a diagnostic setting. Firstly, the biomarker must exist in all strains that the diagnostic test is required to identify. Secondly, the biomarker must not occur in any other organism; i.e. the biomarker must be globally unique to the group of interest in order to avoid false-positive results. Our team inserted a third project specific requirement; the biomarker must encode an epitope on the surface of the cell in order to be accessible with minimal sample preparation. This rationale was inserted, as the biomarker will be more amenable to point of care scenarios if limited sample processing is required.

A custom bioinformatics pipeline building on principles first described by Flanagan and coworkers was exploited in order to produce a set of putative biomarkers in $C$. difficile that conform to the specific selection criteria outlined above ${ }^{13}$. For a more detailed description of the biomarker identification process see Supporting Information.

\section{Bacterial storage, growth and strains used in this work}

All of the following cultures were grown anaerobically at $37^{\circ} \mathrm{C}$ using anaerobic jars and Anaerocult A gas packs (Merck). C. difficile strains were plated on C. difficile agar base (Sigma), with $7 \%$ sheep's blood and C. difficile C.D.M.N. - selective supplement (Oxoid) or cultured in brain heart infusion (BHI) (Sigma) or cooked meat broth (Sigma). Clostridium sordellii, Clostridium perfringens, Peptostreptococcus anaerobius and Enterococcus faecalis were cultured in $\mathrm{BHI}$ or on soy-tryptone agar (15 g/l tryptone, $5 \mathrm{~g} / \mathrm{l}$ soytone, $5 \mathrm{~g} / \mathrm{l} \mathrm{NaCl}, 15 \mathrm{~g} / \mathrm{l}$ agar) supplemented with 7 \% sheep's blood. Clostridium hiranonis was cultured in PY medium (tryptone $5 \mathrm{~g} / \mathrm{l}$, peptone (pepsin digested) $5 \mathrm{~g} / \mathrm{l}$, yeast $10 \mathrm{~g} / \mathrm{l}$, L-cysteine $0.5 \mathrm{~g} / \mathrm{l}$, D-glucose 5 $\mathrm{g} / \mathrm{l})+40 \mathrm{ml}$ salt solution $\left(\mathrm{CaCl}_{2} \cdot \mathrm{H}_{2} \mathrm{O} 0.25 \mathrm{~g} / \mathrm{l}, \mathrm{MgSO}_{4} \times 7 \mathrm{H}_{2} \mathrm{O} 0.5 \mathrm{~g} / \mathrm{l}, \mathrm{K}_{2} \mathrm{HPO}_{4} 1 \mathrm{~g} / \mathrm{l}, \mathrm{KH}_{2} \mathrm{PO}_{4} 1\right.$ $\left.\mathrm{g} / \mathrm{l}, \mathrm{NaHCO}_{3} 10 \mathrm{~g} / \mathrm{l}, \mathrm{NaCl} 2 \mathrm{~g} / \mathrm{l}\right)$. Escherichia coli, Pseudomonas aeruginosa, Citrobacter freundii, Proteus mirabilis and Klebsiella pneumoniae strains were all cultured aerobically at $37^{\circ} \mathrm{C}$ in LB media (bacto-tryptone $10 \mathrm{~g} / \mathrm{l}$, yeast $5 \mathrm{~g} / \mathrm{l}, \mathrm{NaCl} 10 \mathrm{~g} / \mathrm{l}$ ).

Strains used to probe for interaction with mAb521 are shown in Table S-1. The C. difficile Oxford strains $(\mathrm{Ox})$ used in this study are representative members from each of the 12 surface layer types (SLTs) plus an additional strain, Ox575, which contains a hybrid cassette 
comprising the cwp66 gene of cassette 2 and the secA2 and s/pA genes of cassette 6 . The $0 x$ strains were all kindly donated by Dr Kate Dingle, Nuffield Department of Medicine, Oxford. UK.

\section{Monoclonal antibody production}

Based on the identified biomarker amino acid sequence, linear peptides were synthesised to produce mouse mAbs through the hybridoma technique (Abmart).

\section{Hybridoma culture and production of mAb521}

All cell culture reagents were purchased from Sigma. Hybridoma cells specific for mAb521 production were grown at $37^{\circ} \mathrm{C}$ in a $5 \% \mathrm{CO}_{2}$ incubator, in basic media (RPMI 1640, $10 \%$ foetal calf serum (FCS) (v/v), $100 \mathrm{mg} / \mathrm{l}$ penicillin-streptomycin and $2 \mathrm{mM}(\mathrm{w} / \mathrm{v})$ glutamine), initially with the addition of $10 \%(\mathrm{v} / \mathrm{v})$ Condimed to aid recovery. Fully confluent cells were then grown in media with reducing FCS content until they were able to grow efficiently in serum free media ( $10 \%, 5 \%, 2 \%, 0.5 \%$, serum free). Large scale growth of the cells was performed in specialist media, EX-CELL ${ }^{\circledR}$ 610-HSF Serum-Free Medium for Hybridoma Cells, low-protein $(11 \mathrm{mg} / \mathrm{l})$, with L-glutamine, to which $100 \mathrm{mg} / \mathrm{I}$ penicillin-streptomycin was added. Cells were left in this media for 8-10 days until the majority were dead, after which the supernatant was recovered by centrifugation at $13,000 \mathrm{~g}$ for $10 \mathrm{~min}$. mAb521 was purified from the supernatant using a HiTrap Protein G HP prepacked column (GE Healthcare Life Sciences). The column was equilibrated with 10 column volumes buffer $\mathrm{A}(20 \mathrm{mM}$ sodium phosphate $\mathrm{pH}$

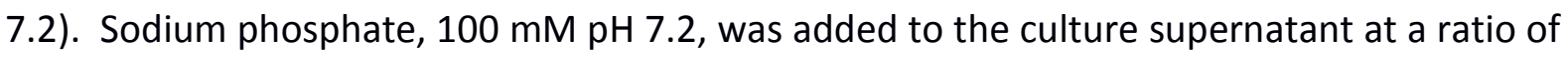
1:5 (final concentration $20 \mathrm{mM}$ sodium phosphate $\mathrm{pH}$ 7.2). A minimum of $260 \mathrm{ml}$ cell culture supernatant was loaded on the column before the application of 20 column volumes of buffer A. Finally, mAb521 was eluted with $100 \mathrm{mM}$ glycine/ $\mathrm{HCl}, \mathrm{pH} 2.3$ in $1 \mathrm{ml}$ fractions collected into $100 \mu \mathrm{l} 1 \mathrm{M}$ Tris- $\mathrm{HCl} \mathrm{pH} 8.0$ to immediately neutralise the fraction.

\section{Polyclonal antibody production against purified surface layer protein A}

Surface layer protein A (SIpA) was purified to homogeneity (see Supporting Information) to raise polyclonal antibodies in mice (Cambridge Research Biochemicals). IgG antibody fractions were recovered from serum using a Protein A column pre-equilibrated in buffer $A$. Briefly, serum was diluted 1 in 5 using $100 \mathrm{mM}$ sodium phosphate $\mathrm{pH} 7.2$ before loading onto a pre-equilibrated Protein $\mathrm{A}$ column. The column was then washed with 10 column volumes of buffer $\mathrm{A}$ before elution using $100 \mathrm{mM}$ glycine/ $\mathrm{HCl}, \mathrm{pH} \mathrm{2.3,} \mathrm{collecting} 1 \mathrm{ml}$ fractions into 100 $\mu \mathrm{I} 1 \mathrm{M}$ Tris- $\mathrm{HCl} \mathrm{pH}$ 8.0. The resulting IgG polyclonal antibody pool was designated pAbSlpA. pAbSIpA was conjugated to HRP using EZ-link Plus Activated HRP (Thermo Fisher) via primary amine groups on the antibody according to manufacturer's instructions. Briefly, $1 \mathrm{mg} p \mathrm{pASSIpA}$ in $50 \mathrm{mM}$ carbonate buffer $\mathrm{pH} 9.6$ was added to lyophilised activated HRP and incubated for $1 \mathrm{~h}$ at room temperature (RT). Thereafter, $10 \mu \mathrm{l} 5 \mathrm{M}$ sodium cyanoborohydride solution (Sigma) was added to the enzyme reaction mix and incubated for $15 \mathrm{~min}$ at RT followed by the addition of $60 \mu \mathrm{l} 1 \mathrm{M}$ ethanolamine $\mathrm{pH} 8.5$ for $15 \mathrm{~min}$ at RT to quench any unreacted material. pAbSIpA-HRP was purified by size exclusion using a HiLoad 16/600 Superdex 200 column, fractions were collected and assessed for antibody binding and HRP activity. 


\section{SPR measurements}

Prior to SPR, mAb521 and SIpA were dialysed extensively into PBS. All analyses of interactions between mAb521 and SIpA were performed on a BIAcore X100 system equipped with a CM5 chip (BIAcore GE Healthcare) at a flow rate of $10 \mu \mathrm{l} / \mathrm{min}$. For immobilization of SIpA, the chip was activated with NHS/EDC (1:1) before injection of SIpA in $10 \mathrm{mM}$ acetate, $\mathrm{pH} 4.0$ to attain 255 Resonance Units (RU) of binding. This is designated the FC2 sample channel. In contrast the FC1 reference channel was NHS/EDC treated and then blocked using ethanolamine. Monoclonal antibody 521 (250 nM-0.65 nM) was injected over both reference and sample surfaces for $300 \mathrm{~s}$ before a $600 \mathrm{~s}$ wait time followed by a 12 $s$ regeneration using $10 \mathrm{mM}$ glycine $\mathrm{pH}$ 2.0. Injection of PBS alone served as a negative control. All measurements were performed in duplicate at $25^{\circ} \mathrm{C}$ in PBS. The results derived from the reference channel FC1 were subtracted from the FC2 sample channel. SPR data was exported and plotted using OriginPro 8. The responses for each of the different antibody concentrations at $380 \mathrm{~s}$ were taken and fitted to a sigmoidal fitting function to derive the apparent $K_{\mathrm{D}}$.

\section{Results and Discussion}

\section{Biomarker identification in C. difficile using a custom bioinformatics pipeline}

The custom bioinformatics pipeline identified 28 biomarker containing proteins that were positive for all surface associated localisation tools (see Supporting Information). Therefore, a total of 28 biomarker containing proteins were predicted to be exposed on the surface of C. difficile. After manual curation and analysis of the 28 biomarker containing proteins, SIpA was judged to be the most promising candidate. The surface layer (S-layer), as the name suggests, typically forms the outermost structure in the bacteria in which they are produced. Functions of the S-layer include cell shape determination, molecular sieving and host cell adhesion/invasion ${ }^{14}$. The proteinaceous $C$. difficile S-layer is encoded by the slpA gene, located within the cell wall protein (cwp) gene cluster, which also includes cwp66 (adhesin) and secA2 (secretory translocase) ${ }^{15}$. Whole genome sequencing and phylogenetic analysis has shown these genes formed a 10-kb cassette, of which 12 distinct surface layer types (SLTs) can be identified ${ }^{16}$. Unlike most bacteria whose S-layers are composed of a single protein subunit, the S-layer of $C$. difficile is constructed using two protein subunits, High Molecular Weight Surface Layer Protein A (HMW SIpA) and Low Molecular Weight Surface Layer Protein A (LMW SIpA) (Figure 1a). The two subunits are derived from a single polypeptide precursor, Surface layer Protein A (SIpA), containing an $\mathrm{N}$-terminal secretion signal ${ }^{17,18}$. The precursor, SIpA, is translocated across the cytoplasmic membrane via the accessory Sec system ${ }^{19}$, which directs the signal peptide across the membrane, where the protein is cleaved by CWP84 (Figure 1b) into the HMW and LMW subunits ${ }^{20}$. The HMW and LMW SIpA form a 1:1 heterodimer complex through non-covalent interactions between highly conserved sequences ${ }^{21}$ in a $\mathrm{Ca}^{2+}$ dependent manner ${ }^{22}$. HMW SIpA contains three tandem cell wall binding 2 motifs (PF04122) which are suggested to be important in mediating interaction with the cell wall, acting as an anchor ${ }^{18}$. These domains interact with anionic polymer PSII, highly conserved in $C$. difficile strains, to direct the protein to the cell wall ${ }^{23}$. It is estimated that there are 590,000 S-layer subunits per cell, requiring synthesis, export and assembly of 164 
subunits per second during exponential growth ${ }^{18,21}$. The S-layer appears to be essential, as evidenced by an inability to generate transposon-mediated insertional mutants within the sIpA gene, consistent with the fact that S-layer proteins have been detected in all $C$. difficile strains to-date ${ }^{21}$. The SIpA biomarker - comprises of 16 amino acids 'AKDGSTKEDQLVDALA' within the HMW subunit. SIpA is the most abundant protein in C. difficile, accounting for 10$15 \%$ of the total cellular protein ${ }^{19}$. Additionally the slpA gene is strongly transcribed during the entire growth phase ${ }^{24}$. These two characteristics suggested SIpA is an ideal diagnostic candidate.

The bioinformatics prediction that the biomarker is surface associated is consistent with the wider literature. In the absence of a high-resolution structure of the $C$. difficile S-layer, it is challenging to predict the precise topology of the S-layer, or the surface accessibility of the HMW biomarker under native anaerobic conditions. LMW SIpA demonstrates higher interstrain variability compared with the HMW subunit ${ }^{25}$, exhibiting on average $32 \%$ sequence identity between any two strains ${ }^{21}$. This suggests a possible role in immune evasion and host cell adhesion. Accordingly, in bacterial adherence studies, testing $C$. difficile adherence to enterocytes, the LMW subunit was found to be more crucial in host-cell attachment than the HMW subunit, suggesting LMW is more surface-exposed ${ }^{26}$. This is also consistent with a HMW: LMW SAXS structure, suggesting HMW is involved in peptidoglycan anchoring ${ }^{21}$. Conversely, evidence also exists that demonstrates the importance of HMW SIpA in adhesion to enterocytes. FACS analysis has shown that antiserum to recombinant HMW completely eliminated $C$. difficile binding to HEp-2 cells and similarly human colon tissue sections incubated with LMW and HMW subunits revealed the HMW subunit showed higher levels of binding 27 .

Regarding the specific 3D location of the biomarker in the HMW subunit, in the absence of a defined crystal structure a suite of bioinformatics tools were used to predict that the biomarker was solvent exposed based on amino acid polarity and secondary structure of the biomarker. A total of $8 \mathrm{mAbs}$ were produced based on the SIpA biomarker sequence and were screened by ELISA for binding to the biomarker peptide sequence and purified SIpA. Two antibodies, mAb521 and mAb652, demonstrated binding to both the peptide sequence and purified SIpA, - mAb521 showed the greatest binding, in all screening assays performed (data not shown) and was taken forward for characterisation.

To be able to perform appropriate laboratory controls to assess the specificity of mAb521 produced against the SIpA biomarker, it was important to identify the species which have amino acid sequences which most closely resemble the SIpA biomarker. BLASTP searches using the SIpA biomarker sequence identified $C$. hiranonis and $P$. anaerobius as having sequences that were the most closely related to SIpA (at the time the BlastP search was performed). However, the avalanche of next generation sequencing data deposited in RefSeq, on a daily basis, makes bioinformatics results highly dynamic and subject to change on a daily basis. A more recent analysis (July 2018) reveals Clostridium argentinense and Clostridium senegalense as the closest SIpA homologs in the form of uncharacterised cell-wall binding repeat containing proteins. That said, the two species chosen are very closely related and still act as exemplars of the reagent specificity. In addition to investigating the similarity of the SIpA sequence to other proteins, the similarity of full genome sequences to $C$. difficile was also explored. The Pathosystems Resource Integration Centre (PATRIC) was used to identify that $C$. sordellii was the species most closely related to $C$. difficile ${ }^{28}$. Thus, $C$. hiranonis, $P$. anaerobius and $C$. sordellii served as negative controls for mAb521 screening where 
appropriate. In addition and possibly more importantly, other bacteria commonly found in stool samples were also screened against the affinity reagents as negative controls.

\section{Western blot analysis against whole cell lysates}

Currently, all sequenced $C$. difficile strains fall into 12 SLTs, which therefore represent the known S-layer diversity within the $C$. difficile species ${ }^{16}$. Whole cell lysates of each of the 12 SLTs plus C. difficile 630 were probed using mAb521 in a Western blot (see Supporting information). Additionally, for completeness, Ox575 was probed for interaction with mAb521 in the event that the hybrid displayed differential binding to mAb521 than any of the 12 SLTs. When analysed by SDS-PAGE, each $C$. difficile cell lysate displayed the characteristic dual banding pattern associated with the presence of HMW SIpA and LMW SIpA (Figure 2). This is in agreement with previous studies which have shown variability in the mobility of the two SLPs between different strains ${ }^{29,30}$. mAb521 (5 $\left.\mathrm{g} / \mathrm{ml}\right)$ binds to HMW SIpA within all 12 SLTs and not to any other proteins within the negative control strains. Furthermore, the positive binding occurs in one defined band, demonstrating unique specificity of mAb521 to HMW SIpA.

\section{Whole cell direct ELISA binding of mAb521 against all known $C$. difficile SLTs}

Whole cells of $C$. difficile 630, the 12 SLTs and Ox575 were tested for interaction with mAb521 $(100 \mathrm{ng} / \mathrm{ml})$ using a whole cell ELISA format (See Supporting Information). The OD600 $\mathrm{nm}$ of the cells was kept constant throughout, enabling semi-quantitative comparative analysis of mAb521 binding. The antibody was shown to recognise all 12 SLTs plus Ox575 whilst displaying negligible cross-reactivity to the closely related species, $C$. hiranonis, $P$. anaerobius and $C$. sordellii (Figure 3 ). Additionally, the binding displayed across the $C$. difficile strains was relatively uniform, with a maximal variation of $35 \%$ between the highest (Ox1437a) and the lowest (Ox1396) absorbance value. If Ox1396 is not considered, the binding will be highly comparable between all remaining SLTs with a variability of $<5 \%$.

Whole cell direct ELISA testing mAb521 against bacteria commonly found in stool samples In order to validate that mAb521 did not cross react with other bacteria typically found in stool samples, a selection of common faecal bacteria were selected and probed for binding to mAb521 using a whole cell ELISA (see supporting information). Agreeably, mAb521 showed limited cross reactivity to all the faecal bacteria tested under these conditions. In fact, the absorbance values were similar to negative controls in which PBS was added instead of cells (Figure 4). C. difficile 630 was used as a positive control and displayed $>90 \%$ binding compared with the highest negative control value of $E$. faecalis.

\section{SPR analysis of mAb521 - SIpA interaction}

To calculate the affinity of mAb521 for its target HMW SIpA, SIpA was purified to homogeneity (See Supporting Information) before being tethered to a CM5 chip. The kinetics of the interaction could not be reliably calculated due to mass transport effects which were consistent under all binding conditions tested (data not shown). As such, the equilibrium dissociation constant for mAb521 binding to SlpA was calculated through duplicate titrations of mAb521 ( $250 \mathrm{nM}-0.65 \mathrm{nM}$ ) over SIpA (Figure 5). The equilibrium dissociation constant for mAb521 binding to SlpA was calculated to be $36.5 \mathrm{nM}$ (Figure 5 inset). This demonstrates that $\mathrm{mAb521}$ can recognise the biomarker when it undertakes its native structure as part of 
SIpA and further validates the whole cell binding assays, which demonstrated universal binding to native protein in all the SLTs.

\section{Sandwich ELISA using mAb521 and pAbSIpA}

Initially we planned to use a sandwich ELISA, using mAb521 for both the capture and detection of whole $C$. difficile cells on the premise that the biomarker is surface exposed, based on the observations from whole cell direct ELISA data (Figures $3 \& 4$ ). Provided the biomarker is indeed surface exposed, whole $C$. difficile cells should be multivalent with respect to the biomarker and therefore should be amenable to capture and detection using mAb521. However, when using a sandwich ELISA format, instead of a whole cell direct ELISA we were unable to detect $C$. difficile cells (data not shown). The simplest explanation for this is that the HMW SIpA biomarker is not surface exposed in anaerobically grown lab cultures of C. difficile 630 and that the whole cell direct ELISAs used to characterise mAb521 binding are detecting SIpA which is exposed or released from $C$. difficile cells during the overnight nonspecific electrostatic adherence to the ELISA plate. However, as the assay conditions are aerobic this could also be a contributing factor in releasing SlpA. Therefore, in order to chemically expose the SIpA biomarker we modified the published glycine extraction method into a rapid 10 min two-step extraction (See Supporting Information). The revised method was applied to various $C$. difficile cell numbers $\left(10^{4} / \mathrm{ml}-10^{9} / \mathrm{ml}\right)$ and purified SIpA before assaying the extract in a sandwich ELISA, using mAb521 for capture and a polyclonal antibody raised against purified SIpA (pAbSIpA-HRP) for detection (see Supporting Information). Using this sandwich ELISA set-up we were able to derive a LOD of $12.4 \mathrm{ng} / \mathrm{ml}$ against purified SIpA (Figure 6a) and detect SIpA released from a minimum of $1.7 \times 10^{6}$ cells $/ \mathrm{ml}$ (Figure 6b).

\section{Conclusions}

This work describes the computational identification of a unique biomarker for $C$. difficile. The biomarker identified by the bioinformatics pipeline was predicted to be conserved in all $C$. difficile strains. This was validated by screening a representative member of each of the known $C$. difficile SLTs against mAb521 in a direct ELISA format in order to assess the specificity of mAb521 independently. This demonstrated mAb521 could, as predicted, bind each of the 12 SLTS of $C$. difficile and, by extrapolation of this representative model data, all C. difficile species. Additionally the biomarker was predicted to be unique to $C$. difficile whilst not being represented in other bacteria. In order to test this,mAb521 was screened using a direct ELISA against a subset of bacteria commonly found in stool samples. As predicted by the bioinformatics pipeline, mAb521 showed minimal cross-reactivity to these faecal bacteria under these conditions. Furthermore, mAb521 displayed negligible cross reactivity to species which have biomarker signatures most closely resembling that of SIpA, $C$. hiranonis and $P$. anaerobius, as well as the most closely related species to $C$. difficile, $C$. sordellii.

This species-specific recognition of $C$. difficile by mAb521 makes it an ideal candidate for a diagnostic antibody. It has been shown on average $\sim 10$-fold more vegetative cells $(\sim 4.75 \mathrm{x}$ $10^{6}$ cells $/ \mathrm{ml}$ ) than spores are typically found in stool samples of patients with CDI ${ }^{31}$. The LOD of $1.7 \times 10^{6}$ cells $/ \mathrm{ml}$, calculated against $C$. difficile cells using our mAb521-pAbSlpA sandwich ELISA demonstrate that it has the capacity to detect $C$. difficile in clinically relevant levels found in stool samples. In order to achieve these clinically relevant levels of detection against 
C. difficile cells, a sample pre-treatment using low $\mathrm{pH}$ glycine was required, taking $10 \mathrm{~min}$. The sample pre-treatment releases SIpA from the S-layer, exposing the SIpA biomarker such that it can interact with mAb521 in its active conformation of a 1:1 heterodimer composed of HMW SIpA : LMW SIpA. Although the HMW biomarker is clearly not accessible on the surface of the bacteria under these aerobic diagnostic assay conditions, there remains the possibility the biomarker is surface exposed under the truly native anaerobic environment of the gastrointestinal tract, so the current data does not completely exclude the possibility of the biomarker being a suitable vaccine or drug target.

The biomarker and antibodies developed are clearly appropriate for centralised laboratory analysis using conventional ELISA formats. The main area of future work will to be apply the biomarker and mAb521 to new assay formats, which are amenable for use at the point of care, such as lateral flow assays enhanced with nanozymes ${ }^{32}$. In addition, we believe it is important to validate the biomarker accessibility in a truly anaerobic model system. Future work will assess mab521 binding to whole $C$. difficile cells under anaerobic conditions. If mAb521 can inhibit $C$. difficile adherence to host enterocytes by neutralising SIpA, the antibody may have potential as a neutralising therapeutic option. Additionally, if the HMW biomarker is found to be surface accessible under anaerobic conditions, this gives the biomarker utility as a vaccination target.

This work demonstrates the bioinformatics approach employed has the potential to locate epitopes ubiquitously expressed across an entire group of interest, whilst mitigating the likelihood of false positive detection by intelligently screening potential biomarkers against RefSeq data for "other non-target bacteria". From this work it is clear the biomarker "AKDGSTKEDQLVDALA" is a universal species identifier, which was successfully used to guide the production of a universal antibody, mAb521, against the species, C. difficile. In addition, mAb521, in conjunction with a polyclonal partner has both high sensitivity and specify to the biomarker. A clinical study is planned in order to evaluate mAb521 detection of SIpA, as a direct comparator to the GDH EIA, in a two-step testing algorithm for $C$. difficile.

The computational approach described here for biomarker identification could also be deployed against any Gram-positive or Gram-negative bacteria with sequences deposited in RefSeq. The team are pursuing further confirmatory targets in both categories.

\section{Associated Content}

The Supporting Information is available free of charge on the

ACS Publications website at DOI:

Computational and experimental methods, bacterial strains used in this work and bioinformatics approach to biomarker identification. 


\section{Acknowledgements}

This research was funded by the EPSRC IRC in Early-Warning Sensing Systems for Infectious Diseases (i-sense) EP/K031953/1. We would like to thank Dr Kate Dingle for the kind donation of the Oxford $C$. difficile strains.

\section{Citations}

(1) Warny, M.; Pepin, J.; Fang, A.; Killgore, G.; Thompson, A.; Brazier, J.; Frost, E.; McDonald, L. C. Lancet 2005, 366, 1079-1084.

(2) Sp Moore, L.; Donaldson, H. Br. J. Hosp. Med. (Lond.) 2013, 74 Suppl 10, C146-149.

(3) Wilkins, T. D.; Lyerly, D. M. J. Clin. Microbiol. 2003, 41, 531-534.

(4) Casari, E.; De Luca, C.; Calabro, M.; Scuderi, C.; Daleno, C.; Ferrario, A. Antimicrob. Resist. Infec. Control. 2018, 7, 40.

(5) Polage, C. R.; Gyorke, C. E.; Kennedy, M. A.; Leslie, J. L.; Chin, D. L.; Wang, S.; Nguyen, H. H.; Huang, B.; Tang, Y. W.; Lee, L. W.; Kim, K.; Taylor, S.; Romano, P. S.; Panacek, E. A.; Goodell, P. B.; Solnick, J. V.; Cohen, S. H. JAMA Intern. Med. 2015, 175, 1792-1801.

(6) $\mathrm{DOH}$. Updated guidance on the diagnosis and reporting of clostridium difficile, 2012.

(7) O'Connor, J. R.; Johnson, S.; Gerding, D. N. Gastroenterology 2009, 136, 1913-1924.

(8) Brazier, J. S.; Raybould, R.; Patel, B.; Duckworth, G.; Pearson, A.; Charlett, A.; Duerden, B. I. Euro Surveill. 2008, 13.

(9) Wilcox, M. H.; Shetty, N.; Fawley, W. N.; Shemko, M.; Coen, P.; Birtles, A.; Cairns, M.; Curran, M. D.; Dodgson, K. J.; Green, S. M.; Hardy, K. J.; Hawkey, P. M.; Magee, J. G.; Sails, A. D.; Wren, M. W. Clin. Infect. Dis. 2012, 55, 1056-1063.

(10) Tenover, F. C.; Baron, E. J.; Peterson, L. R.; Persing, D. H. J. Mol. Diagn. 2011, 13, 573-582.

(11) Fang, F. C.; Polage, C. R.; Wilcox, M. H. J. Clin. Microbiol. 2017, 55, 670-680.

(12) Tenover, F. C.; Novak-Weekley, S.; Woods, C. W.; Peterson, L. R.; Davis, T.; Schreckenberger, P.; Fang, F. C.; Dascal, A.; Gerding, D. N.; Nomura, J. H.; Goering, R. V.; Akerlund, T.; Weissfeld, A. S.; Baron, E. J.; Wong, E.; Marlowe, E. M.; Whitmore, J.; Persing, D. H. J. Clin. Microbiol. 2010, 48, 37193724.

(13) Journal of integrative bioinformaticsFlanagan, K.; Cockell, S.; Harwood, C.; Hallinan, J.; Nakjang, S.; Lawry, B.; Wipat, A. J. Integr. Bioinform. 2014, 11, 242.

(14) Sara, M.; Sleytr, U. B. J. Bacteriol. 2000, 182, 859-868.

(15) Sebaihia, M.; Wren, B. W.; Mullany, P.; Fairweather, N. F.; Minton, N.; Stabler, R.; Thomson, N. R.; Roberts, A. P.; Cerdeno-Tarraga, A. M.; Wang, H.; Holden, M. T.; Wright, A.; Churcher, C.; Quail, M. A.; Baker, S.; Bason, N.; Brooks, K.; Chillingworth, T.; Cronin, A.; Davis, P., et al. Nat. Genet. 2006, 38, 779-786.

(16) Dingle, K. E.; Didelot, X.; Ansari, M. A.; Eyre, D. W.; Vaughan, A.; Griffiths, D.; Ip, C. L.; Batty, E. M.; Golubchik, T.; Bowden, R.; Jolley, K. A.; Hood, D. W.; Fawley, W. N.; Walker, A. S.; Peto, T. E.; Wilcox, M. H.; Crook, D. W. J. Infect. Dis. 2013, 207, 675-686.

(17) Calabi, E.; Ward, S.; Wren, B.; Paxton, T.; Panico, M.; Morris, H.; Dell, A.; Dougan, G.;

Fairweather, N. Mol. Microbiol. 2001, 40, 1187-1199.

(18) Kirk, J. A.; Banerji, O.; Fagan, R. P. Microb. Biotechnol. 2017, 10, 76-90.

(19) Fagan, R. P.; Fairweather, N. F. J. Biol. Chem. 2011, 286, 27483-27493.

(20) Kirby, J. M.; Ahern, H.; Roberts, A. K.; Kumar, V.; Freeman, Z.; Acharya, K. R.; Shone, C. C. J. Biol. Chem. 2009, 284, 34666-34673.

(21) Fagan, R. P.; Albesa-Jove, D.; Qazi, O.; Svergun, D. I.; Brown, K. A.; Fairweather, N. F. Mol. Microbiol. 2009, 71, 1308-1322. 
(22) Takumi, K.; Endo, Y.; Koga, T.; Oka, T.; Natori, Y. Tokushima J. Exp. Med. 1992, 39, 95-100.

(23) Ganeshapillai, J.; Vinogradov, E.; Rousseau, J.; Weese, J. S.; Monteiro, M. A. Carbohydr. Res. 2008, 343, 703-710.

(24) Savariau-Lacomme, M. P.; Lebarbier, C.; Karjalainen, T.; Collignon, A.; Janoir, C. J. Bacteriol. 2003, 185, 4461-4470.

(25) Calabi, E.; Fairweather, N. J. Bacteriol. 2002, 184, 3886-3897.

(26) Merrigan, M. M.; Venugopal, A.; Roxas, J. L.; Anwar, F.; Mallozzi, M. J.; Roxas, B. A.; Gerding, D. N.; Viswanathan, V. K.; Vedantam, G. PLoS One 2013, 8, e78404.

(27) Calabi, E.; Calabi, F.; Phillips, A. D.; Fairweather, N. F. Infect. Immun. 2002, 70, 5770-5778.

(28) Snyder, E. E.; Kampanya, N.; Lu, J.; Nordberg, E. K.; Karur, H. R.; Shukla, M.; Soneja, J.; Tian, Y.; Xue, T.; Yoo, H.; Zhang, F.; Dharmanolla, C.; Dongre, N. V.; Gillespie, J. J.; Hamelius, J.; Hance, M.; Huntington, K. I.; Jukneliene, D.; Koziski, J.; Mackasmiel, L., et al. Nucleic Acids Res. 2007, 35, D401406.

(29) Br J Hosp Med (Lond)Cerquetti, M.; Molinari, A.; Sebastianelli, A.; Diociaiuti, M.; Petruzzelli, R.; Capo, C.; Mastrantonio, P. Microb. Pathog. 2000, 28, 363-372.

(30) Takeoka, A.; Takumi, K.; Koga, T.; Kawata, T. J. Gen. Microbiol. 1991, 137, 261-267.

(31) Jump, R. L.; Pultz, M. J.; Donskey, C. J. Antimicrob. Agents Chemother. 2007, 51, 2883-2887.

(32) Loynachan, C. N.; Thomas, M. R.; Gray, E. R.; Richards, D. A.; Kim, J.; Miller, B. S.; Brookes, J. C.; Agarwal, S.; Chudasama, V.; McKendry, R. A.; Stevens, M. M. ACS Nano 2018, 12, 279-288.

\section{TOC graphic}

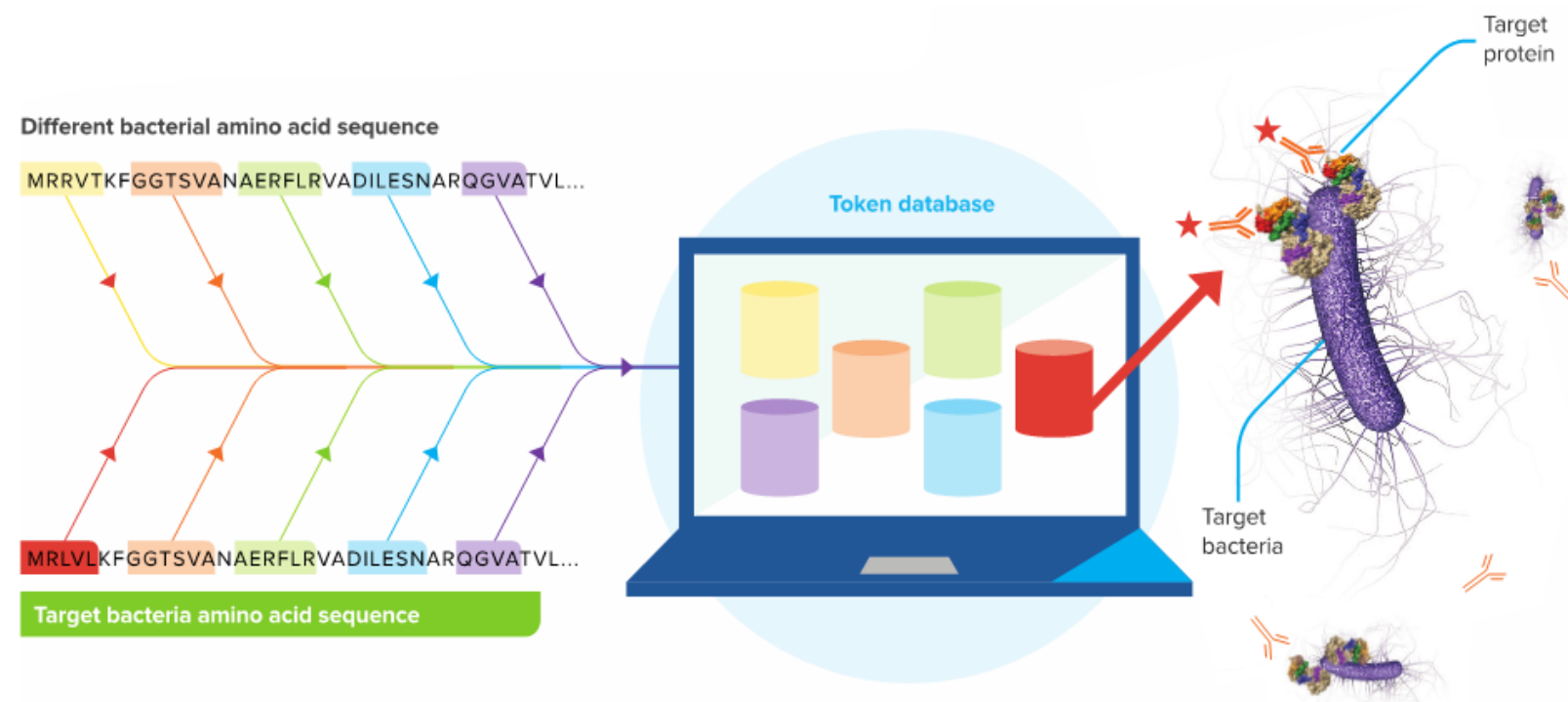




\section{Figures}

(a)

(b)

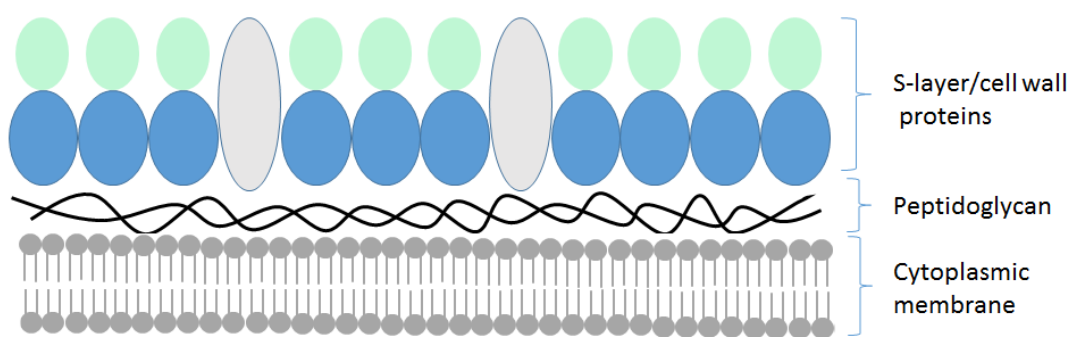

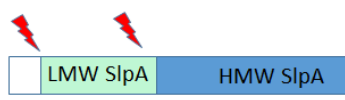

Figure 1: Schematic representation of the $C$. difficile cell wall and SIpA processing. (A) $C$. difficile cell wall - the two SLPs are shown, HMW SIpA (blue) and LMW SIpA (green). Other minor cell wall proteins are shown as ovals (grey). (b) The precursor protein SIpA showing the cleavage sites (lightning bolt) generating the signal peptide (white rectangle), the mature LMW SIpA (green rectangle) and HMW SIpA (blue rectangle). 

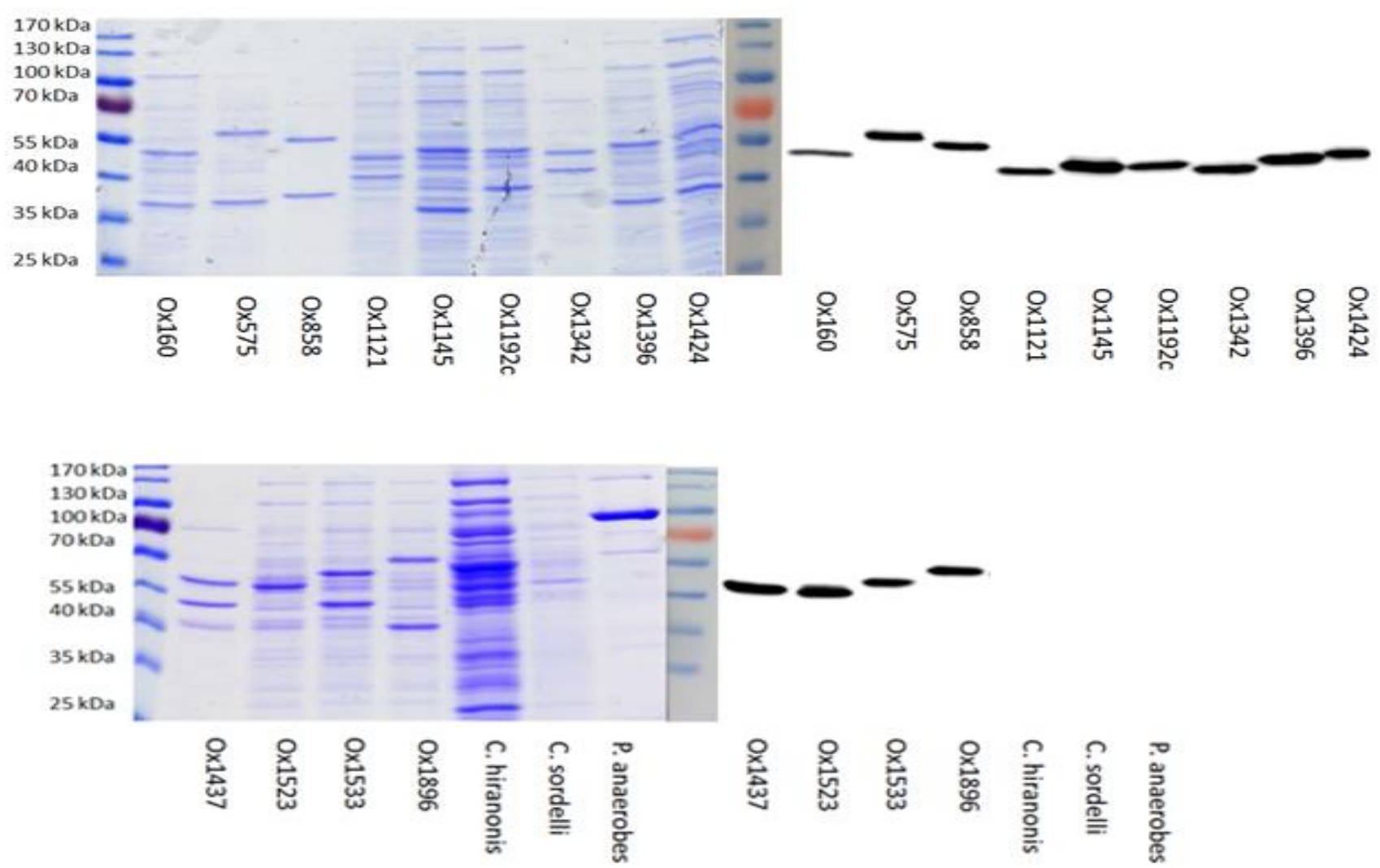

Figure 2: Western blot analysis against whole cell lysates: mAb521 $(5 \mu \mathrm{g} / \mathrm{ml})$ was tested for binding against whole cell lysates from the 12 SLTs, Ox575 and the closely related species C. hiranonis, $C$. sordellii, and P. anaerobius.

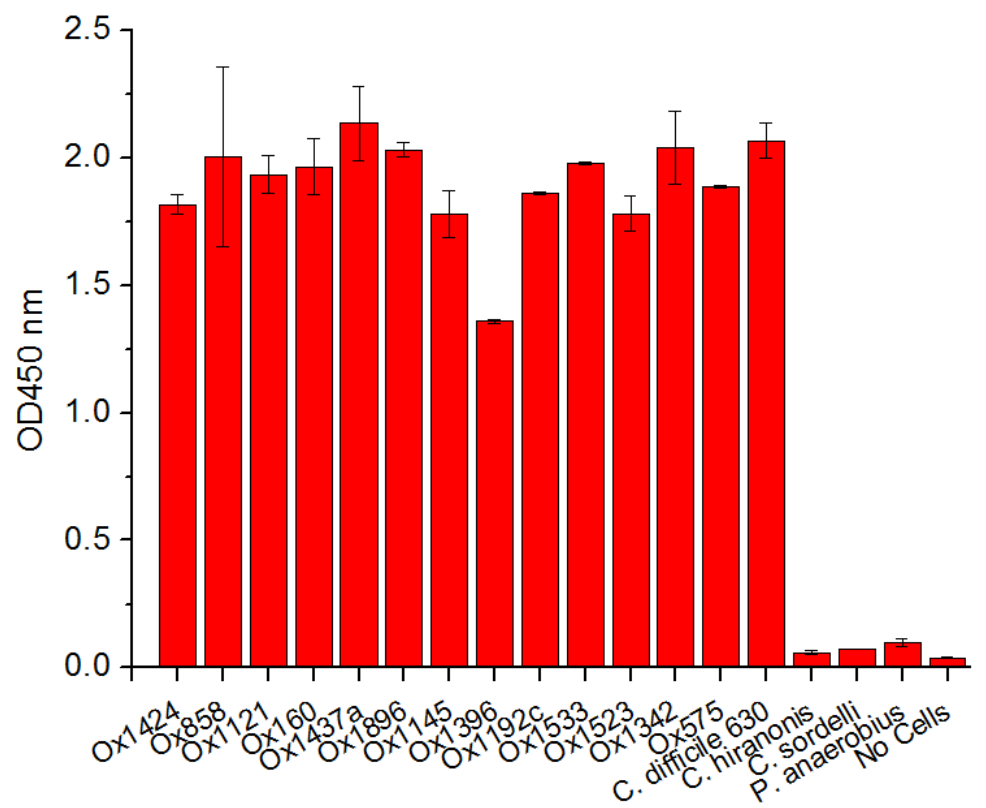

Figure 3: Whole cell direct ELISA using mAb521 against all SLTs: mAb521 (100 ng/ml) was probed for binding against the 12 SLTs, Ox575 and the closely related species $C$. hiranonis, $C$. sordellii, and $P$. anaerobius. The error bars show the standard deviation seen between the two replicates of each sample. 


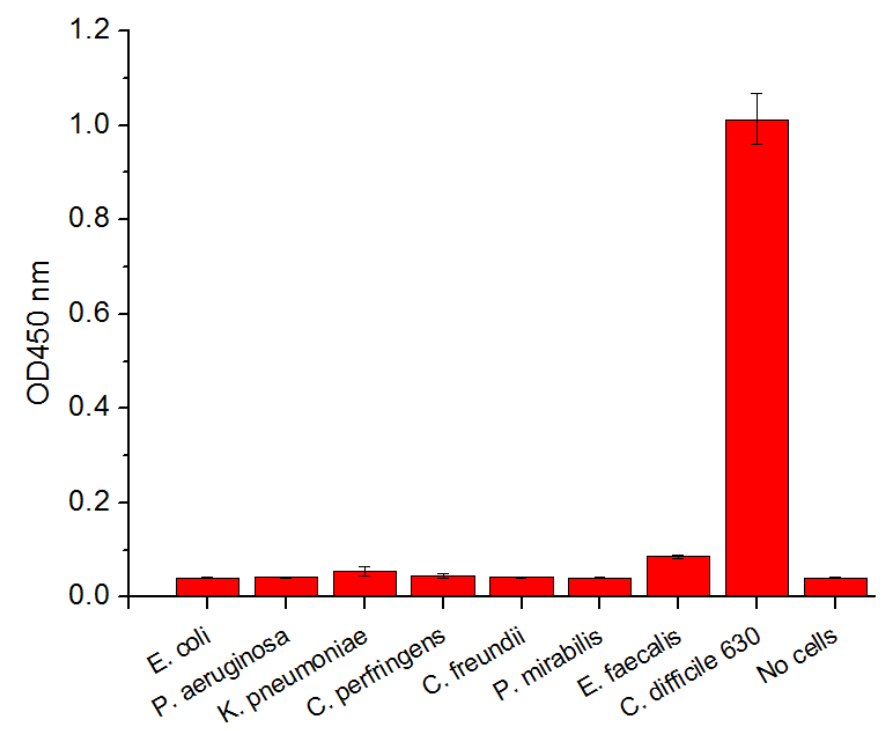

Figure 4: Whole cell direct ELISA using mAb521 against faecal bacteria. mAb521 $(100 \mathrm{ng} / \mathrm{ml})$ was probed for binding against the common faecal bacteria Escherichia coli, $P$. aeruginosa, K. pneumoniae, C. perfringens, $C$. freundii, $P$. mirabilis and E. faecalis. C. difficile 630 was used as a positive control and PBS as a negative control. The error bars show the standard deviation seen between the two replicates of each sample.

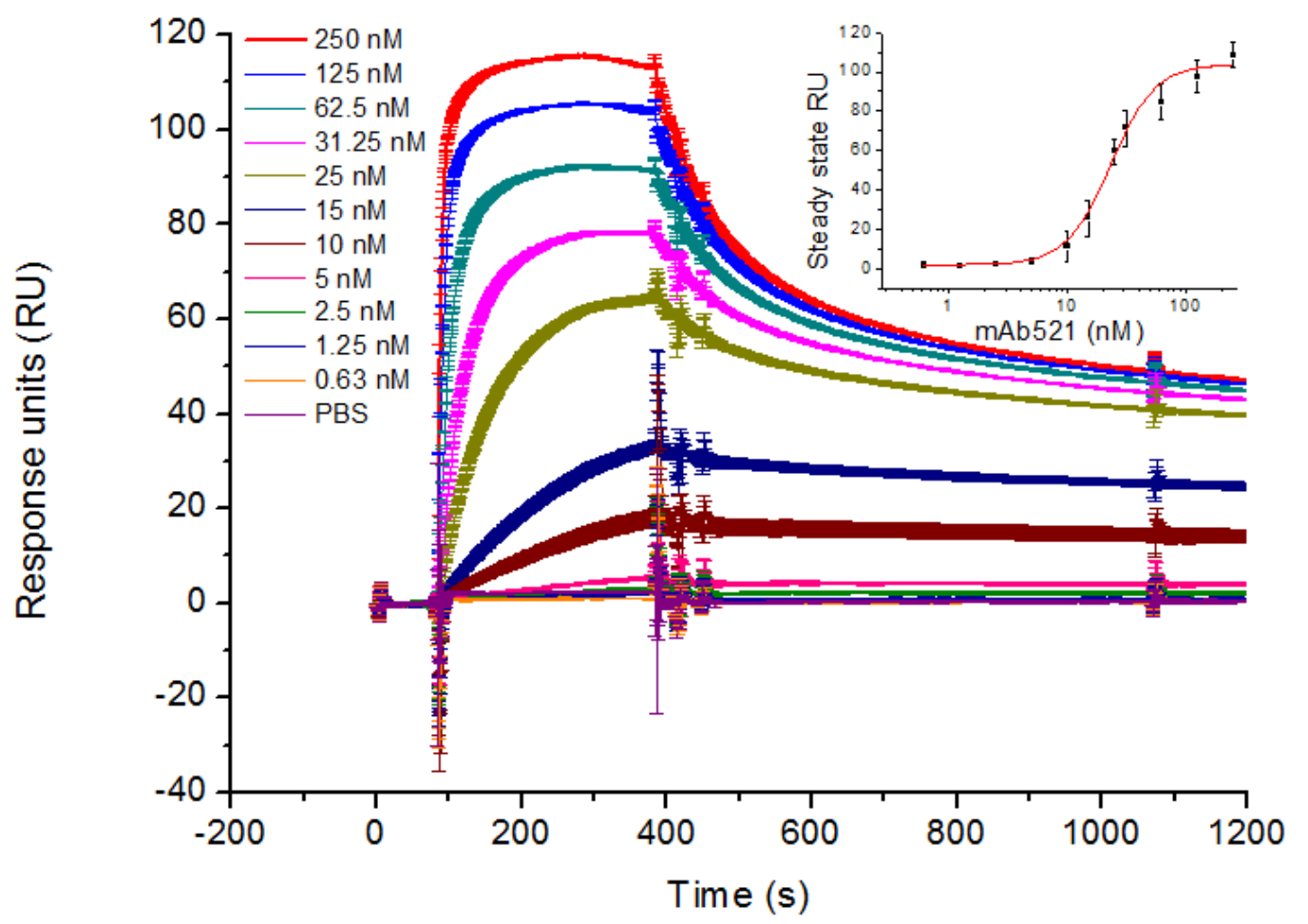


Figure 5: Using SPR to characterise the binding of mAb521 against purified SIpA. The apparent equilibrium dissociation constant $\left(K_{\mathrm{D}}\right)$ for $\mathrm{mAb5} 21$ binding to SIpA was calculated at $380 \mathrm{~s} . \mathrm{R}^{2}=0.99$ (inset) through duplicate titrations of mAb521 ( $250 \mathrm{nM}-0.65 \mathrm{nM}$ ) over SlpA (main figure). Duplicate injections of PBS over SIpA served as negative controls. The error bars show the standard deviation seen between the two replicates of each sample.

(a)

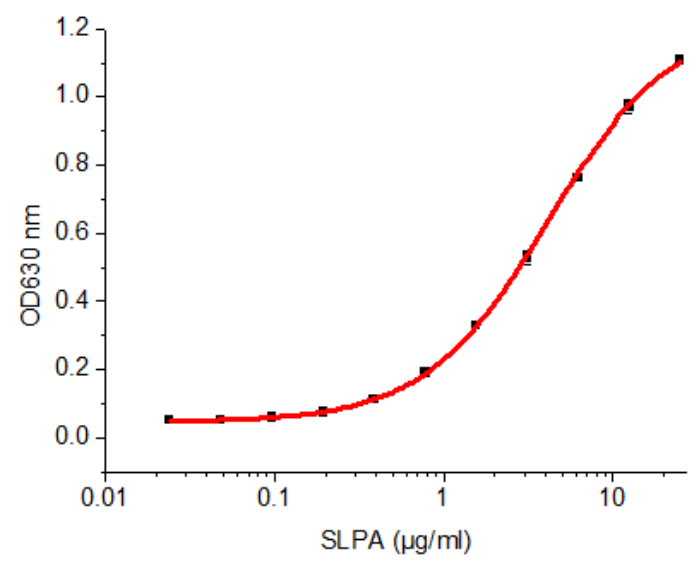

(b)

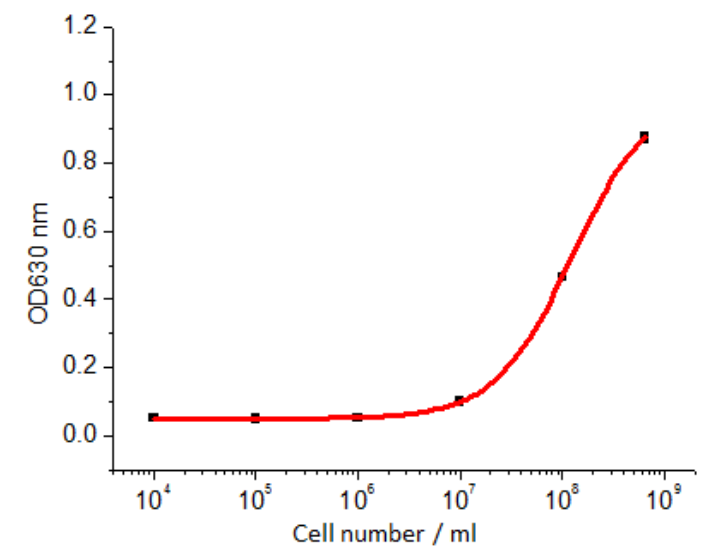

Figure 6: Determining the LOD of the sandwich ELISA. (a) Sandwich ELISA against purified SlpA using mAb521/pAbSIpA. $R^{2}=0.99$. (b) Sandwich ELISA against rapid glycine extracted cells $\left(10^{9}-10^{4} / \mathrm{ml}\right)$, extracted for $10 \mathrm{~min} . \mathrm{R}^{2}=0.99$. The error bars show the standard deviation seen between the two replicates of each sample. 